\title{
Intraply crack and delamination interaction in laminate beams under transverse loading
}

\author{
Y.-B. Shi \& A. F. Yee \\ Department of Materials Science and Engineering, University of Michigan, Ann Arbor, Michigan 48109-2136, USA
}

\begin{abstract}
Intraply cracking and delamination are the basic modes of damage in composite laminates and their interaction constitutes an important phase in damage development. Such an interaction is investigated in the present work through a two-dimensional finite element analysis of a three-point bending beam model. This configuration is considered to embody the basic characteristics of lowenergy transverse impact of composite laminates, where damage is a major concern. Interleaves are incorporated in the model to evaluate their role in the damage process. The stacking sequence used is $[0 / \mathrm{A} / 90 / \mathrm{A} / 0]$ (' $\mathrm{A}$ ' denotes an interleaf), and an intraply crack at $45^{\circ}$ to the loading line is assumed in the central $90^{\circ} \mathrm{ply}$. The damage process is simulated by specifying the delamination growth at the tips of the intraply crack along the beam axis direction. Stress fields and the strain energy release rates are evaluated at different stages of the delamination growth.

The results show that before any delamination takes place, there is a significant transverse normal stress concentration at the tips of the intraply crack. Consequently, there is a strong involvement of mode I component of fracture at this stage. However, with the growth of the delamination, a rapid transition occurs in which the transverse normal stress and its corresponding mode I delamination driving force decrease dramatically relative to their shear counterparts. The entire damage process is therefore summarized as primarily a mode II delamination fracture dominated event preceded by a very limited period of mode I driven fracture at the initiation of the delamination by the intraply crack. The results also indicate that soft interleaves do not alleviate the fracture driving forces at the delamination front. It follows that the interleaf technology relies almost entirely on the toughness of the interleaves.
\end{abstract}

\section{INTRODUCTION}

\subsection{Interaction between intraply crack and delamination}

A major design concern in structural applications of high performance fiber composite materials is their susceptibility to damage. Intraply cracking (splitting along fibers) and delamination have been identified as the most basic modes of damage, and are known to interact to form a three-dimensional damage network. These two modes have been analyzed individually; their interaction under various laminate loading conditions, however, has not been adequately studied. With recent development in toughening composites for improved damage tolerance, there exists a pressing demand for more precise knowledge regarding the interaction between intraply crack- ing and delamination. Within this context, a model analysis is conducted in the present work to examine such an interaction.

Experimental studies have suggested that there are two intraply cracking and delamination interaction situations in a laminate panel subject to out-of-plane impact, i.e. (a) delamination initiation by a bending-related tensile intraply crack at the back surface, and (b) delamination initiation by a transverse shear-related intraply crack inside the laminate. These are shown in Fig. 1. Investigations into the first situation have been reported, ${ }^{1,2}$ and the delamination has been concluded to be predominantly mode I fracture. The second situation has only been examined recently, ${ }^{3}$ and the results show a significant through-thickness normal stress concentration, implying the involvement of mode I component of fracture in further delamination growth. On the other hand, a delamination analy- 
sis model $\mathrm{l}^{4,5}$ as shown in Fig. 2, which is believed to resemble the second interaction situation, predicts pure mode II delamination fracture. It is therefore of interest to investigate in detail the fracture modes of delamination, from its initiation to propagation, in association with the intraply crack.

\subsection{Interleafing technology}

One effective toughening technology for composite laminates with better tolerance to out-ofplane impact is the incorporation of tough homogeneous ${ }^{6}$ or particulate-filled ${ }^{7,8}$ interleaves into interlaminar regions. Experimental evidence ${ }^{9}$ has revealed that the introduction of interleaves dramatically reduces the extent of delamination under impact. In addition, the presence of interleaves alters, in some circumstances, the resulting geometry of impact damage, ${ }^{10}$ as schematically shown in Fig. 3. One question has to be answered in order to rationalize and optimize the interleafing technology; namely, what are the physical mechanisms pertinent to the observed reduction of damage and alteration of damage geometry? Recent investigations ${ }^{11,12}$ relevant to this question have concluded that the ability of the interleaves to undergo plastic deformation under the constraint of the adjacent plies reflects the measured

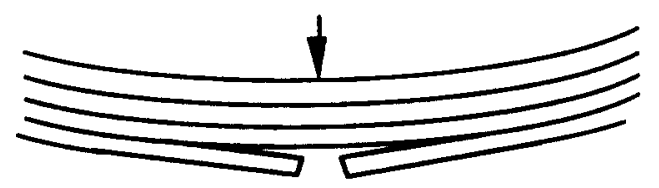

(a)

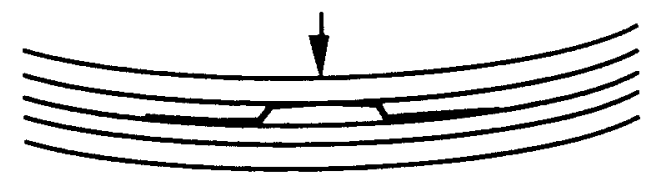

(b)

Fig. 1. Two situations of intraply crack and delamination interaction in laminates with low-energy impact damage. (a) Interaction at back surface; (b) interaction inside laminate.

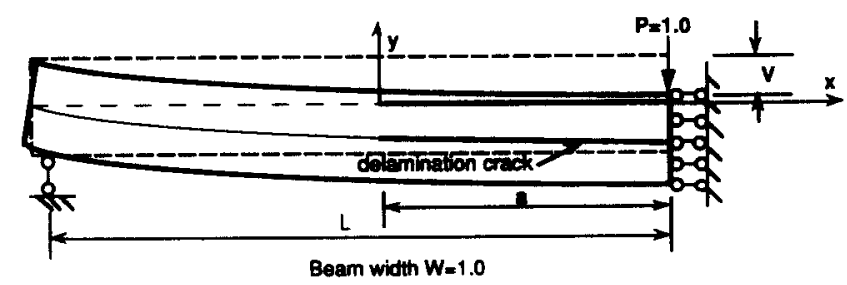

Fig. 2. Delamination model simulating delamination propagation in laminates under impact load. apparent toughness of the interleaved materials during delamination propagation. To the present authors' knowledge, the effect of interleaves on the stress distribution and fracture driving forces remain to be fully explained.

\subsection{Objective of present work}

The present study chooses to analyze both the initiation of delamination by an intraply crack and its further propagation under transverse load, which bears similarity to the impact damage process of laminates. For the ease of analysis, a three-point bending type of loading geometry is adopted, as shown in Fig. 4 . The primary objective of the analysis is to identify the fracture modes at different stages of the damage evolution and see how they are affected by the interleaves. Stress field and its corresponding strain energy release rates are the key parameters examined.

\section{PROBLEM STATEMENT AND FINITE ELEMENT MODEL}

In the model shown in Fig. 4, the stacking sequence of the laminate beam is $[0 / \mathrm{A} / 90 / \mathrm{A} / 0]$, where ' $A$ ' denotes an interleaf. The thickness of the interleaves was varied to facilitate the examination of its effect on the crack propagation driving forces. The total height of the beam was maintained when the thickness of the interleaf was changed. This was achieved through adjusting the thicknesses of the $0^{\circ}$ and $90^{\circ}$ sublaminates. Table 1 shows the combinations of the thickness values

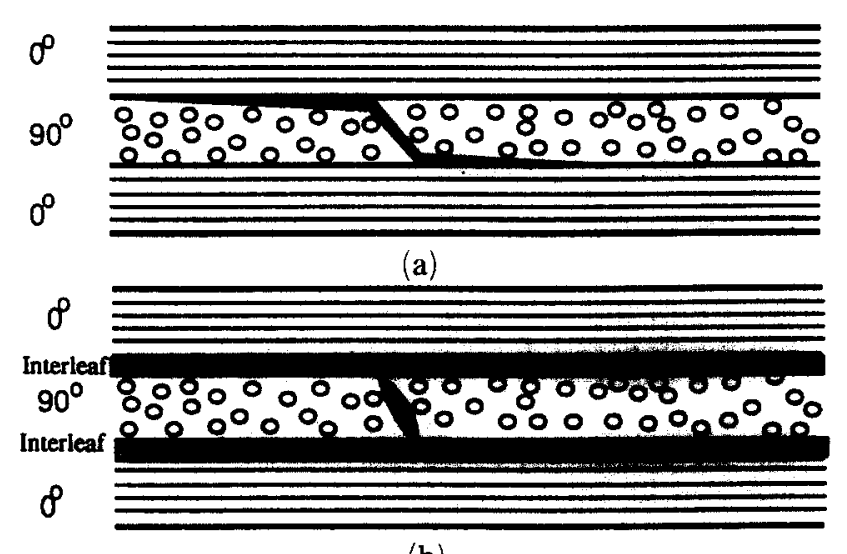

(b)

Fig. 3. Schematic illustration of alteration of impact damage geometry by interleaves. (a) Delamination and intraply cracks always connected in non-interleaved laminate; (b) intraply crack existing independent of delamination in interleaved laminate. 
of the interleaves and the sublaminates in the computations.

While it is possible to determine exactly the location of the intraply crack by analyzing the contact stress field generated by the transverse load and employing an appropriate strength criterion, the amount of computational work thus incurred would greatly restrict the ensuing modeling of the delamination mechanism in great detail, which is the major factor of concern in this study. As a result of this consideration, the existence of an intraply crack at $45^{\circ}$ to be beam axis is presumed in the $90^{\circ}$ sublaminate, as shown in Fig. 4. It is noted that this assumption is based on experimental observations and is in agreement with the result of a similar model analysis. ${ }^{3}$ Given the fact that the current model focuses only on the aspect of interaction between intraply crack and delamination, such an assumption appears to be justifiable.

The material properties of the composite and the interleaves are chosen to resemble those of typical carbon/epoxy composites and thermoplastic interleaves. These are given in Table 2.

The finite element discretization is shown in Fig. 5. A plane-strain condition in the $x-y$ plane is assumed. The actual computation can be divided into two stages, i.e. (1) the initiation of delamination from the intraply crack (i.e. $b=0$ ); and (2) the propagation of delamination along the lower interface $\left(b=b_{1}, b_{2}, b_{3}, b_{4}\right.$ and $\left.b_{5}\right)$. In the following, results of these two stages are presented

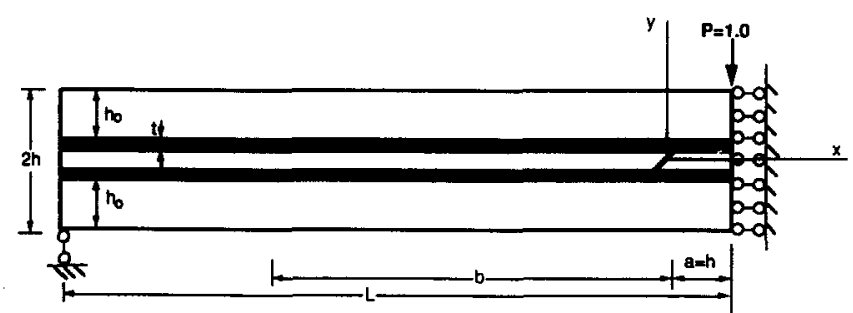

Fig. 4. Three-point bending type model for analysis (beam width $W=1 \mathrm{~mm}$, height $2 h=2.5 \mathrm{~mm}$, length $L=22.5 \mathrm{~mm}$ ). (Only half of the model is shown due to symmetry. Diagram not plotted to scale for clarity). separately. All delaminations were assumed to occur in the middle of the interleaves (or at the $0 / 90$ and $90 / 0$ interfaces when no interleaf was present). Since only linear elastic analysis was conducted, the applied load and the width of the beam were assumed to be unity $(P=1.0 \mathrm{~N}$ and $W=1.0 \mathrm{~mm})$. The finite element mesh generation was carried out using the PATRAN package, and the actual analysis using the ABAQUS package.

\section{DELAMINATION INITIATION}

As a first step in the delamination initiation study, an intraply crack was the only damage assumed in the model. Stress fields at both ends $(x=-0 \cdot 25$, $y=-0.25 \mathrm{~mm}$ and $x=0.25, y=0.25 \mathrm{~mm}$, see Fig. 4 ) of the intraply crack were examined. An example of the computational results is given in Figs $6(\mathrm{a})$ and (b), where through-thickness normal stress $\left(\sigma_{y}\right)$ and transverse shear stress $\left(\tau_{x y}\right)$ for interleaf thickness $t_{3}$ are plotted along the axial direction of the beam in the middle of the interleaf. As expected, stress concentration exists at both ends of the transverse crack. An important feature of the stress distribution is that $\sigma_{y}$ in front of the intraply crack along the beam axial direction are tensile, at both tips of the intraply crack. Furthermore, the ranges of stress elevation are larger in front of the crack than behind.

Figures $7(\mathrm{a})$ and (b) give comparisons of stress distribution under different interleaf thicknesses at the lower end of the intraply crack $(x=-0 \cdot 25$, $y=-0.25 \mathrm{~mm}$ ). Both transverse normal and shear stress distributions are seen to be influenced by the interleaf thickness. For the normal stress $\sigma_{y}$, the far-field value approaches zero, thus allowing a direct comparison of the stress profiles. It is seen, from Fig. 7(a), that the range of stress elevation decreases with increasing interleaf thickness. For the transverse shear stress, a thicker interleaf leads to a higher far-field value (note that the transverse load was kept unchanged at unity). To facilitate a more cogent comparison, data for different interleaf thicknesses in Fig. $7($ b) are

Table 1. Interleaf thickness values and corresponding sublaminate heights

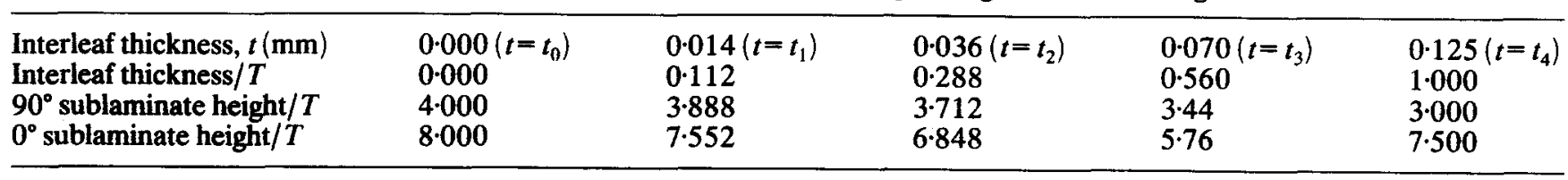

Note: $T=0.125 \mathrm{~mm}$ is the thickness of one individual composite ply. 
Table 2. Material properties used in finite element computations

\begin{tabular}{lccccccc}
\hline & $\begin{array}{c}E_{11} \\
(\mathrm{GPa})\end{array}$ & $\begin{array}{c}E_{22} \\
(\mathrm{GPa})\end{array}$ & $\begin{array}{c}G_{12} \\
(\mathrm{GPa})\end{array}$ & $\begin{array}{c}G_{13} \\
(\mathrm{GPa})\end{array}$ & $\mu_{12}$ & $\mu_{13}$ \\
\hline $\begin{array}{l}\text { Composite plies } \\
\text { Interleaves }\end{array}$ & $140 \cdot 0$ & $10 \cdot 0$ & 6.0 & $6 \cdot 0$ & 0.34 & 0.34 \\
\hline
\end{tabular}

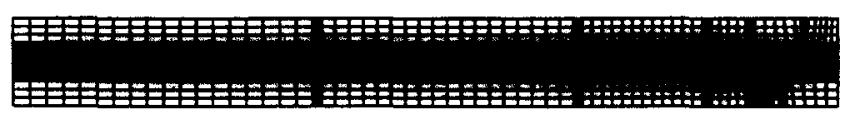

Fig. 5. Illustration of the finite element model used in the computations.

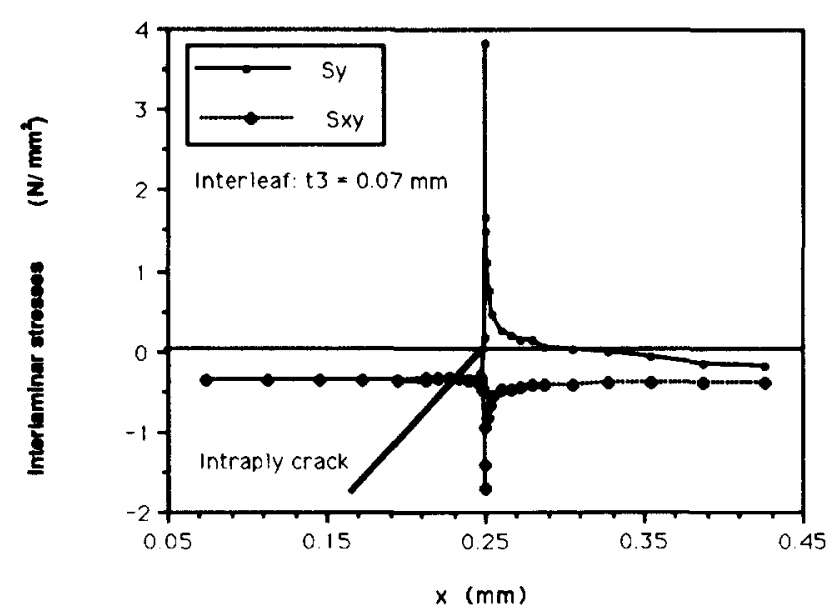

(a)

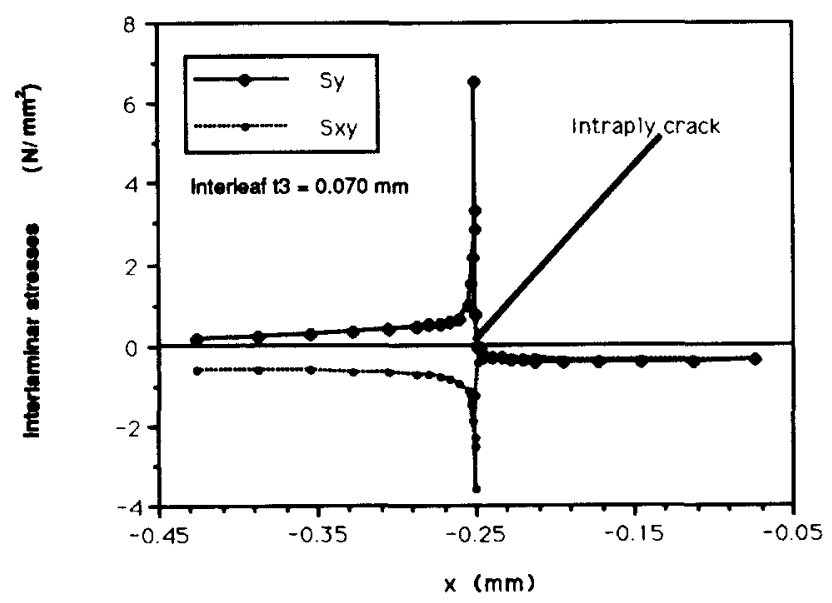

(b)

Fig. 6. Stress distributions at intraply crack tips. (a) Upper tip of intraply crack; (b) lower tip of intraply crack.

normalized by the far-field value approximated by the last data point of each curve. The result is illustrated in Fig. 8. It becomes apparent that the actual stress concentration for the interlaminar shear stress is almost identical under different

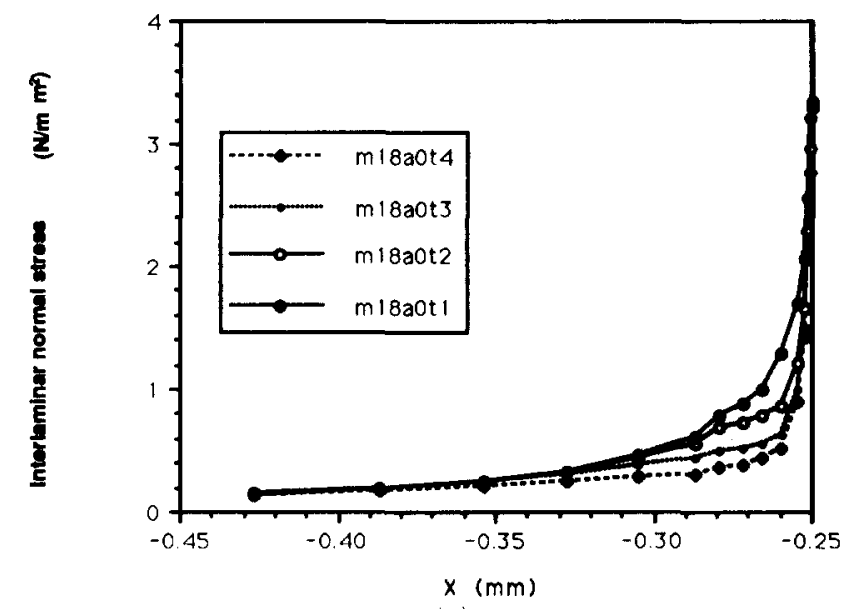

(a)

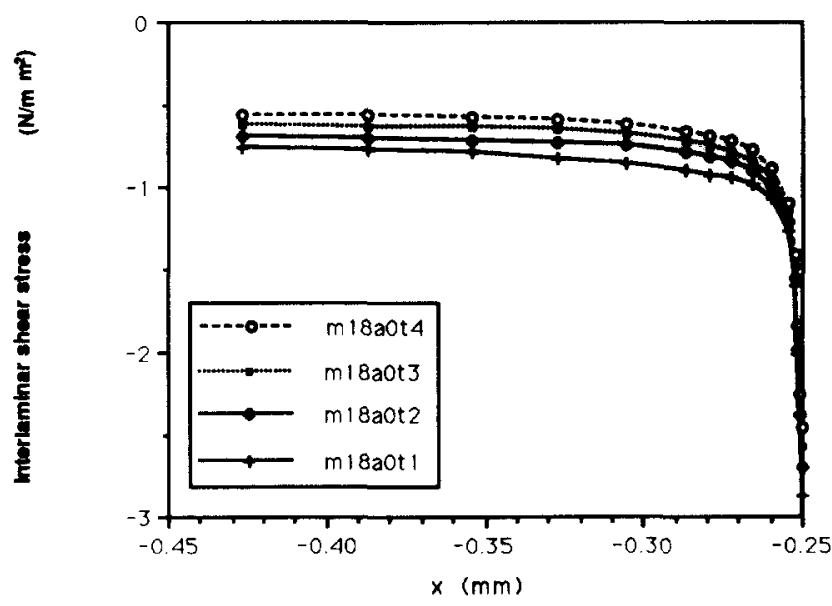

(b)

Fig. 7. Influence of interleaves on stress distribution at lower intraply crack tips $(y=-0.25 \mathrm{~mm})$. (a) Interlaminar normal stress; (b) interlaminar shear stress.
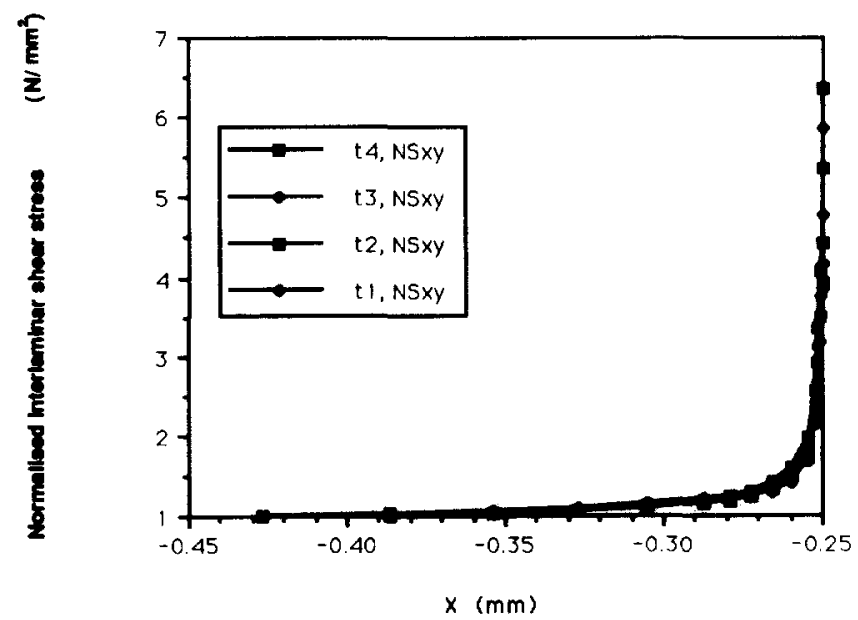

Fig. 8. Normalized distribution of interlaminar shear stress at lower intraply crack tip $(y=-0.25 \mathrm{~mm})$.

interleaf thickness. The data in Fig. 8 are in the close vicinity of the crack tip which is enclosed by a homogeneous interleaf, and therefore the stress profiles should be dominated by the inverse 
square root singularity predicted by linear fracture mechanics. To pursue this point further, crack tip stresses are plotted in logarithmic scales as shown in Fig. 9. It is seen that approaching the

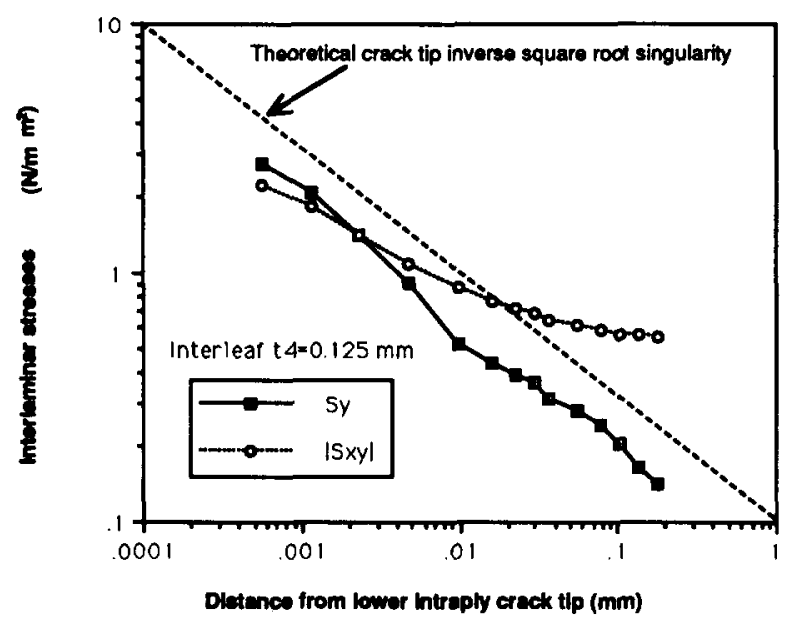

Fig. 9. Comparison of theoretical and numerical predictions of crack tip near-field stress distribution.

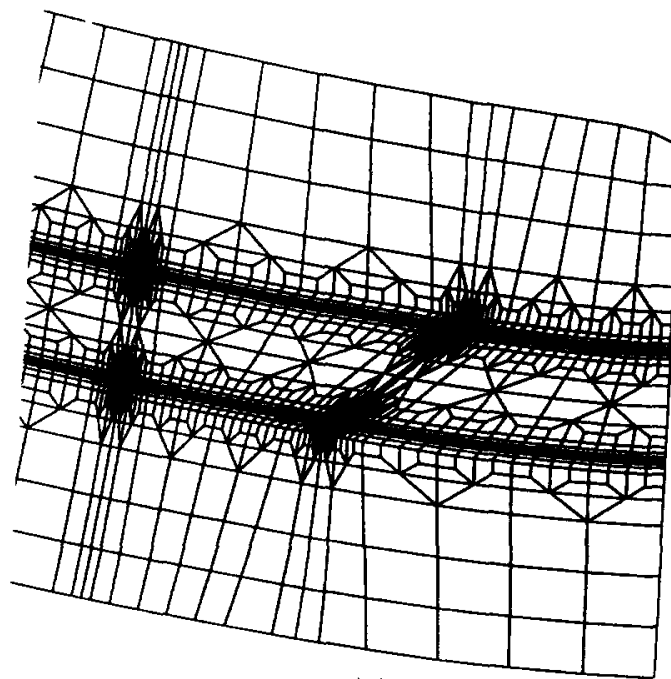

(a)

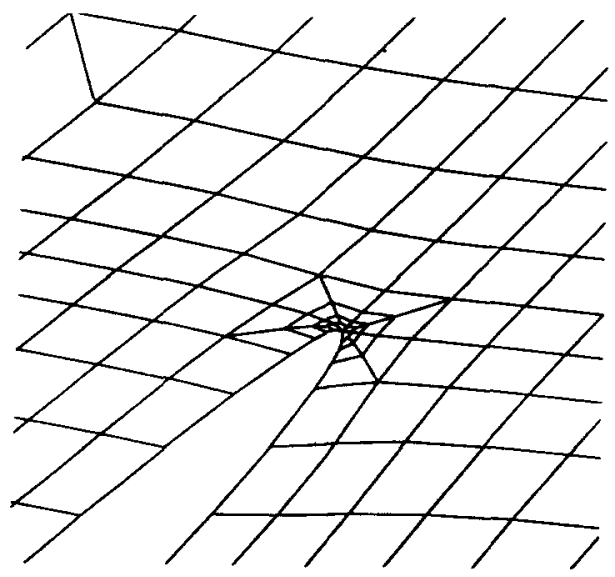

(c) crack tip, the stresses indeed appear to follow the theoretical slope represented by the straight line.

The co-existence of both interlaminar normal and shear stress components indicates that delamination initiation by the intraply crack parallel to the plies would be mixed mode fracture. It is then of interest to know, from a fracture mechanics point of view, the relative magnitude of the two components of the strain energy release rate (SERR). To achieve this, the virtual crack closure technique by Rybicki ${ }^{13}$ was employed to decompose the SERR. Two series of computations are discussed in the following. Figure 10 shows the deformed configurations of the tip regions of the intraply crack from the two series. In both series, an incremental delamination initiation was assumed at the lower end of the intraply crack $(x=-0.25, y=-0.25 \mathrm{~mm})$ along the negative $X$ axis direction. The two series differ at the upper end of the intraply crack $(x=0.25, y=0.25 \mathrm{~mm})$

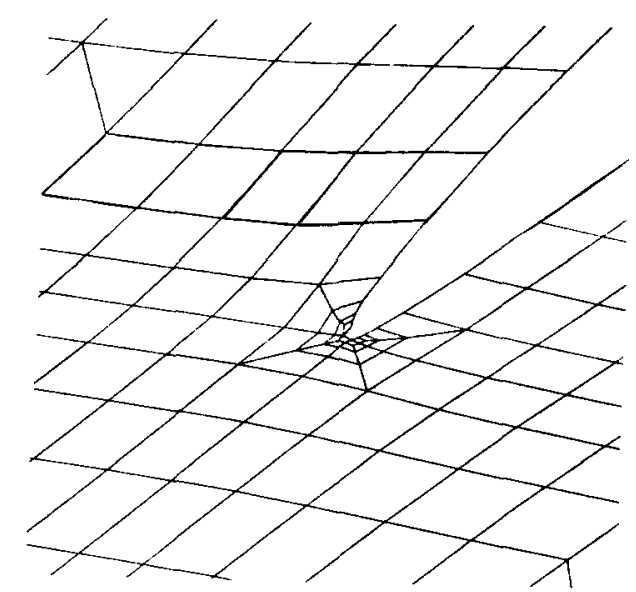

(b)

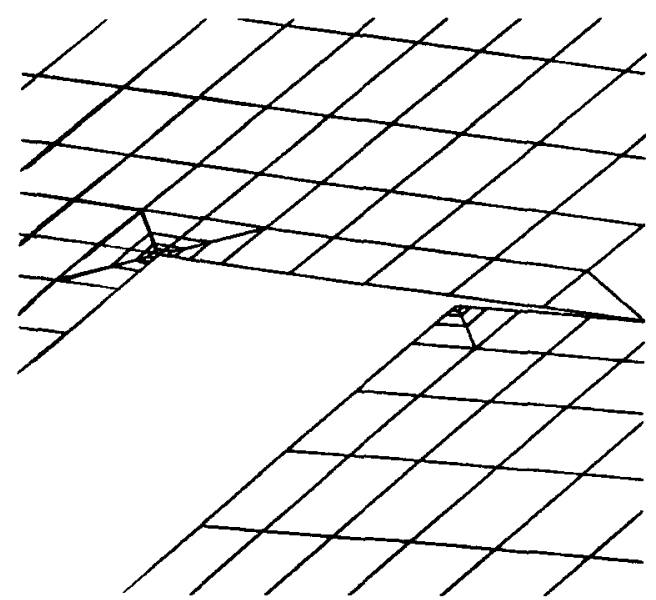

(d)

Fig. 10. Deformed configurations of intraply crack tip regions. (a) Overall view of intraply crack region; (b) lower intraply crack tip, one incremental node release simulating delamination initiation; (c) upper intraply crack tip without delamination; (d) upper intraply crack tip with complete delamination at upper interface. 
where in the first no crack extension was allowed (Fig. 10(b)), whereas in the second a complete delamination was assumed to have formed at the upper end of the intraply crack (Fig. 10(c)). The ratios of the two components of the SERR in the two series of computations are given in Fig. 11. It

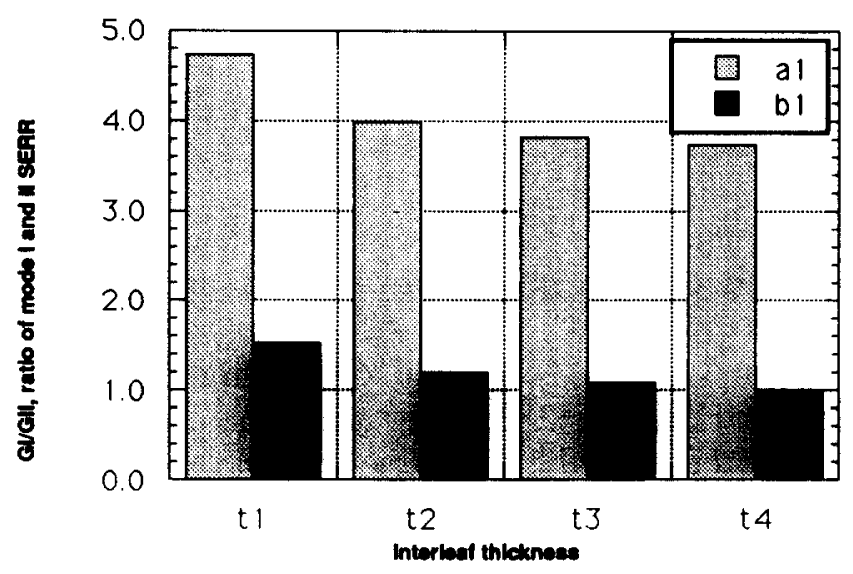

Fig. 11. Ratio of mode I and II components of SERR (series $a_{1}$ : no delamination at intraply crack tips, i.e. combination of Figs $10(\mathrm{~b})$ and $(\mathrm{c})$; series $b_{1}$ : complete delamination at upper interface and no delamination at lower interface, i.e. combination of Figs $10(b)$ and $(d))$. is seen that at delamination initiation, there is a strong involvement of mode I fracture. This substantiates the stress field results presented in the last paragraph. It is also interesting to note that the pre-existence of the delamination at the upper shoulder of the intraply reduces the mode I to II ratio quite significantly.

\section{DELAMINATION PROPAGATION}

In the second stage of the computation, the focus of attention was on delamination growth at the lower interface $(y=-0.25 \mathrm{~mm})$. Results of the stress fields and the SERRs corresponding to five delamination lengths $b=b_{1}$ to $b_{5} \quad\left(b_{1}=0.25\right.$, $b_{2}=1.25, b_{3}=2.50, b_{4}=6.25$ and $b_{5}=13.75 \mathrm{~mm}$; for definition of $b$ see Figs 4 and 5). In all the computations presented, complete delamination was assumed in the middle of the upper interleaf (i.e. $0 \cdot 25 \leq x \leq 1.25$ and $y=0.25 \mathrm{~mm}$ ).

A general impression of the stress distribution at the delamination front can be obtained from Fig. 12 which shows the stress contours for the case of $b=b_{5}$. A particular point to note is that,
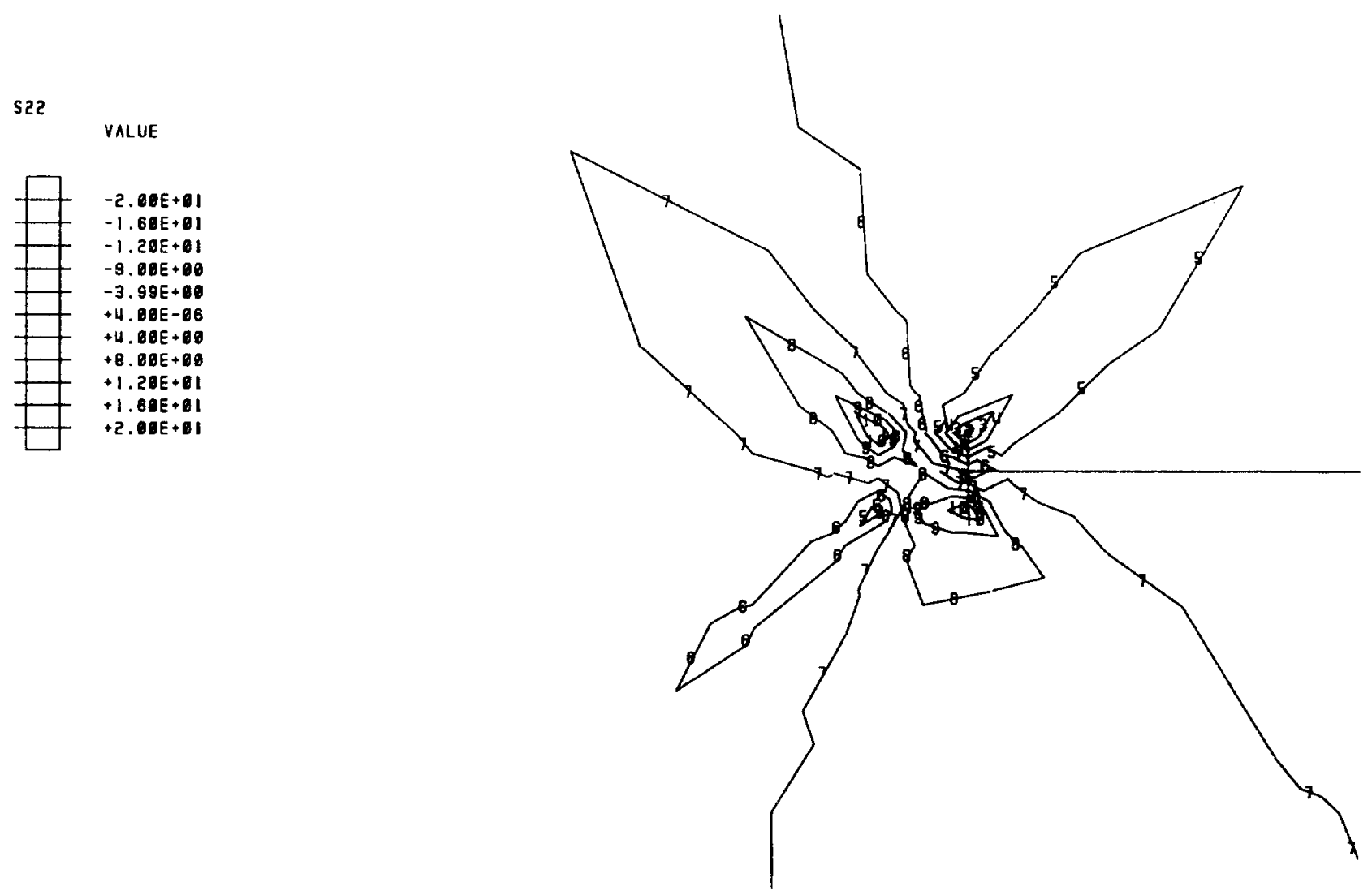

(a)

Fig. 12. Stress contours at delamination crack tip $\left(b=b_{5}\right)$. (a) Interlaminar normal stress; interlaminar shear stress; (c) von Mises stress. 
s12

vaLUE
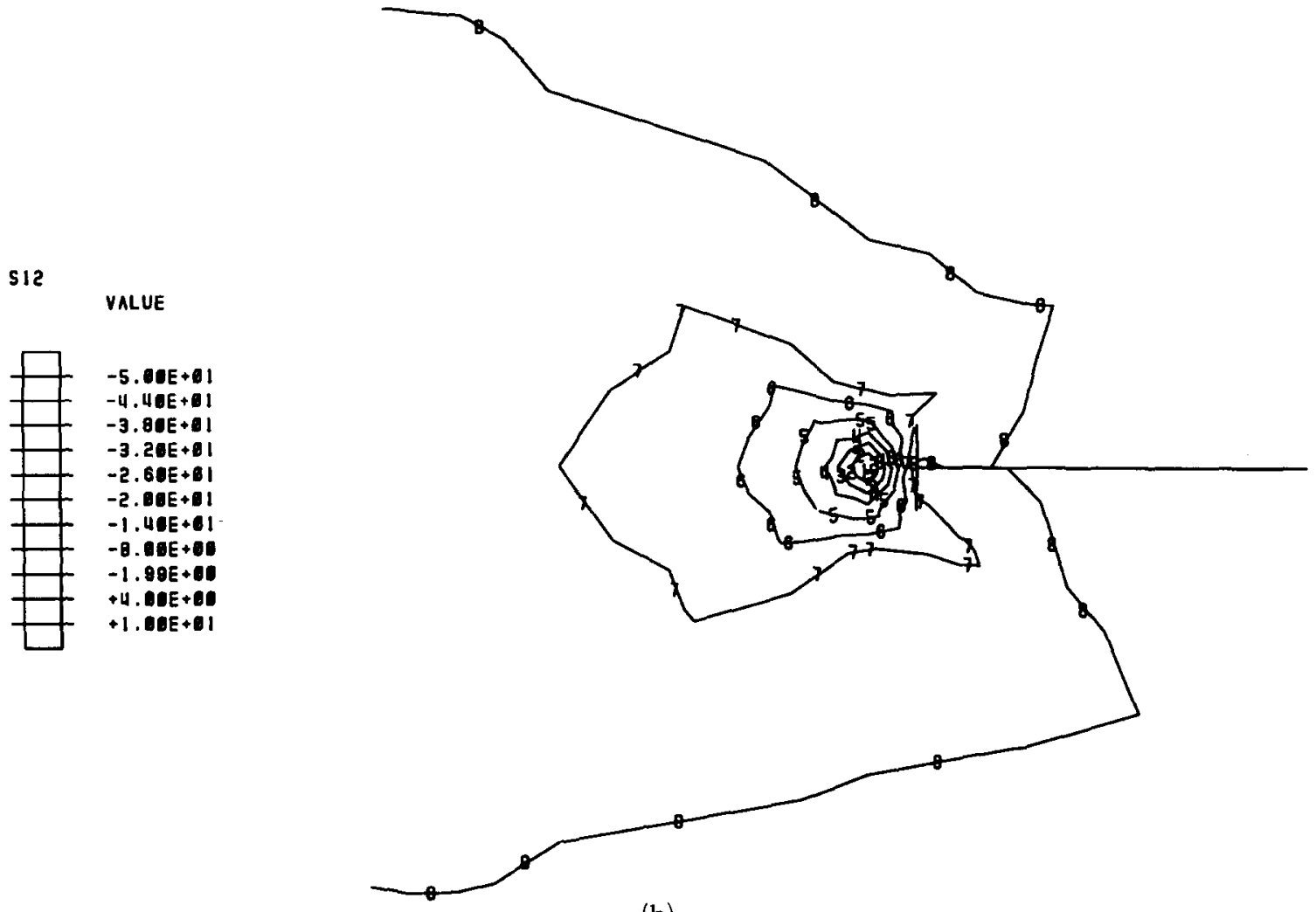

(b)
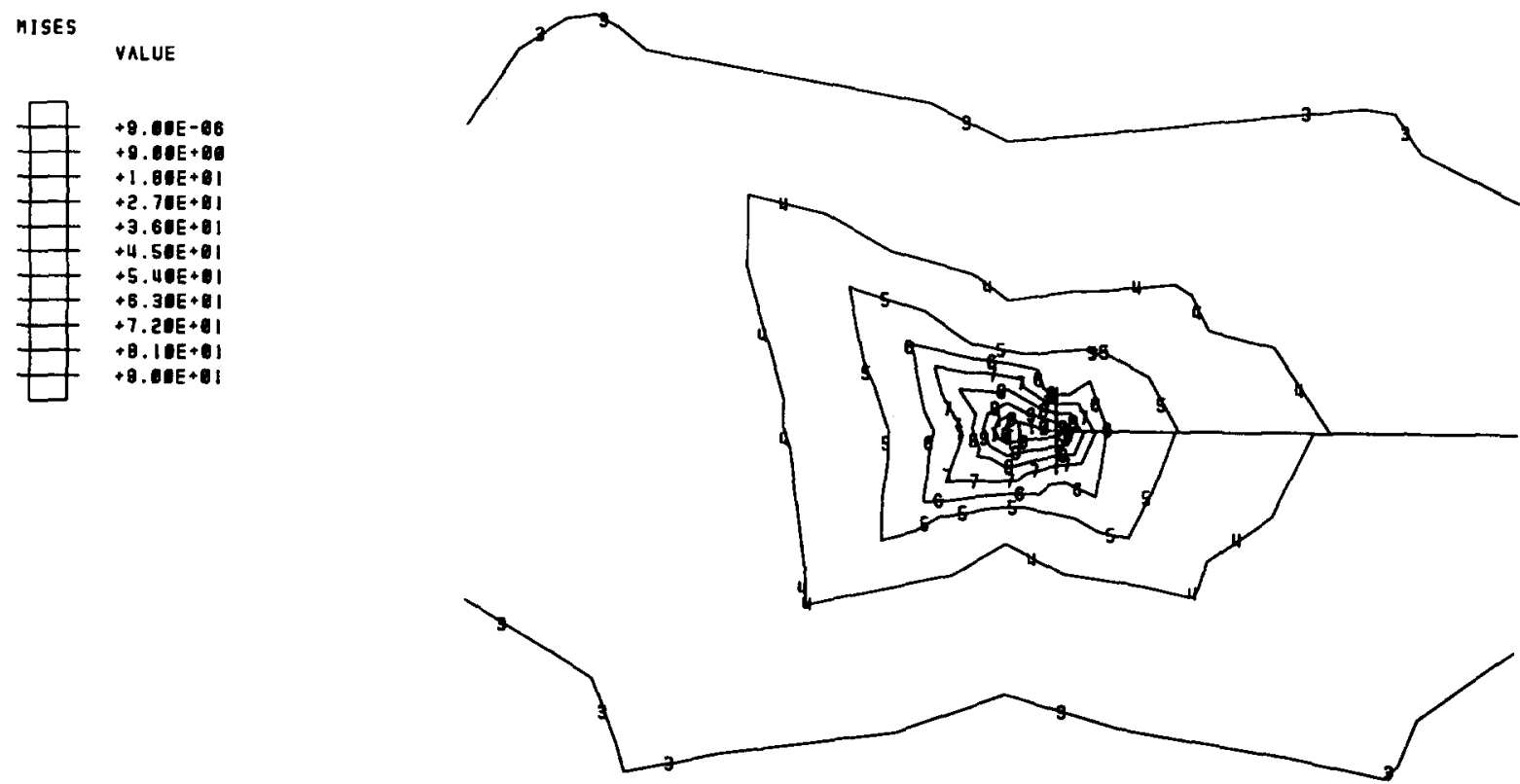

(c)

Fig. 12. - contd.

unlike the crack initiation cases introduced in Section 3, no through-thickness normal stress concentration is preset at the crack tip. Only that of the interlaminar shear stress remains.

Figure 13 gives a representative example of SERR results with an interleaf thickness of $t_{2}$. Both mode I and mode II components increase with the length of the lower delamination (i.e. $b$ ).
More importantly, immediately after the initial growth of the delamination, the mode II component outweighs the mode I component. In fact this is true for all the interleaf thickness values investigated, as shown in Fig. 14 (for clarity, $t_{0}$ case is omitted; see Fig. 10 for this case). Figure 15 shows the sum of mode I and II SERRs obtained from the virtual crack closure approach. The total 


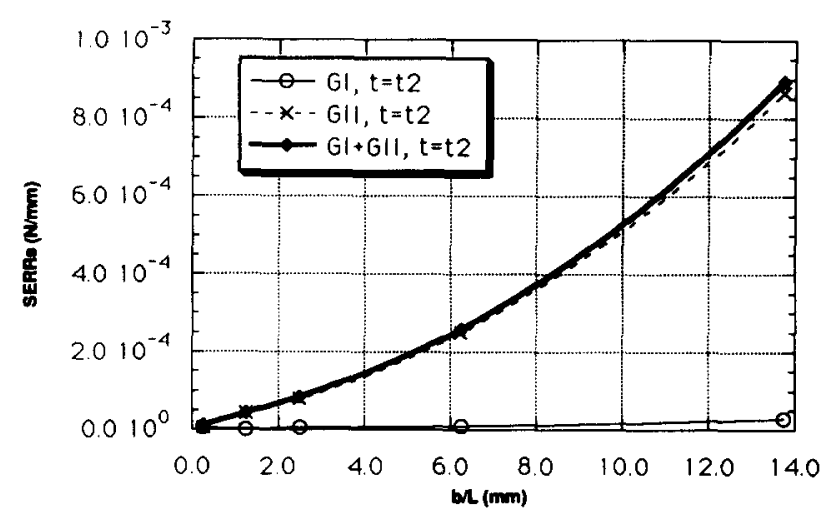

Fig. 13. Example of variation of SERRs with delamination length (complete delamination at upper interface).

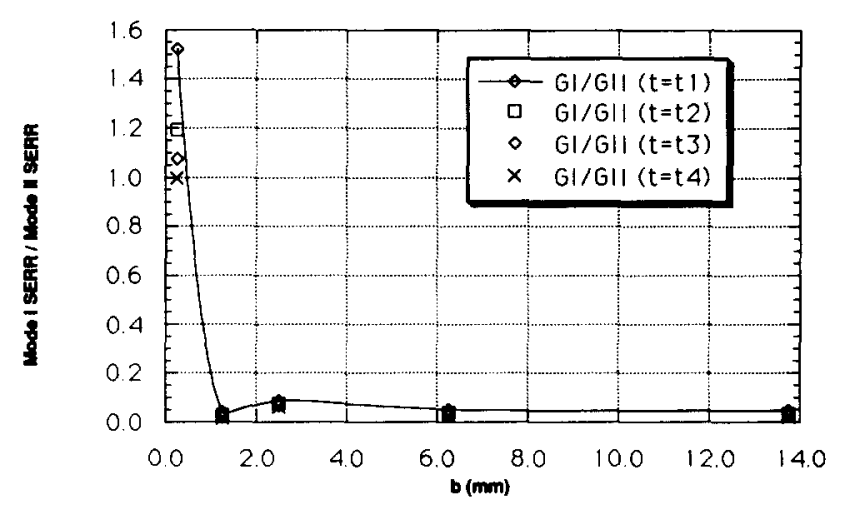

Fig. 14. Ratio of mode I and II components of SERR at different delamination lengths (complete delamination at upper interface).

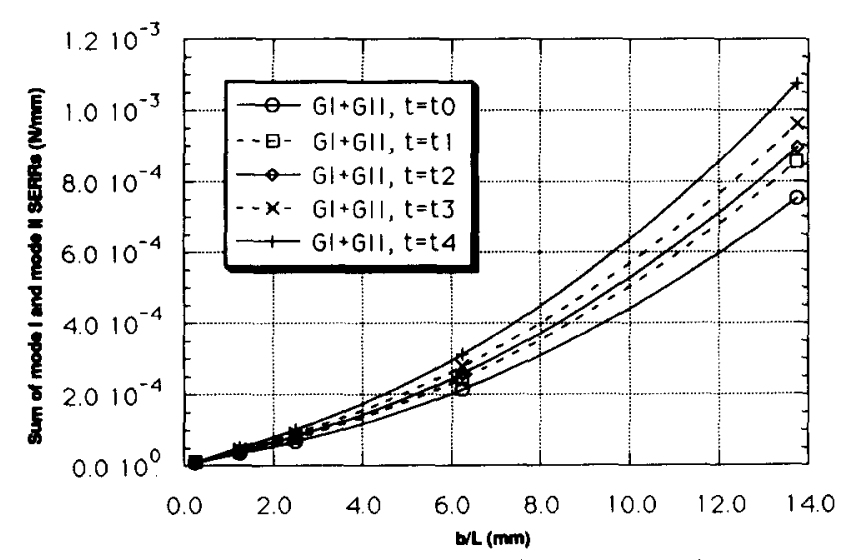

Fig. 15. Variation of total SERR with delamination length.
SERR of the model was also computed using the compliance differential (CD) approach, the result of which should constitute a rather rigorous independent reference to the results shown in Figs 13-15. Table 3 presents an example of the comparison of SERR results by the virtual crack closure and the compliance differential approaches. Excellent agreement is found between the results by the two approaches, indicating the good accuracy of the virtual crack closure mode partition.

Figure 16 can be used to assess the effect of the interleaves from another perspective. At the initiation of the lower delamination (Fig. 16(a)), an increase in the thickness of the interleaves is seen to reduce the mode I and promotes the mode II SERR, bringing the values of the two components closer. The same effect is observed in all the other delamination lengths investigated, except that under these circumstances, mode II SERR dominates.

From Fig. 16, the total SERR at a given delamination length is seen generally to increase with the thickness of the interleaves. However, as in the case of the interlaminar shear stress, the effect of the constant load should be eliminated to examine the effect of the interleaves on the crack driving forces. The present study examines a parameter, the strain energy release rate per unit total strain energy (SERR/SE). This proposed parameter appears to be more meaningful and conceptually clearer than the SERR when the fracture driving forces of two different structures are compared. it is in fact the amount of energy released relative to the stored elastic energy for a unit area crack extension. From the viewpoint of stress distribution, it reflects the stress concentration of a structure. Figure 17 plots SERR/SE against the interleaf thickness for the models in this study. Based on this figure, an argument can be made

Table 3. Comparison of strain energy release rate evaluation by virtual crack closure (VCC) and compliance differential (CD) approaches ( $b=b_{1}$ as an example)

\begin{tabular}{|c|c|c|c|c|c|c|}
\hline $\begin{array}{l}\text { Interleaf } \\
\text { thickness }\end{array}$ & $\begin{array}{c}\text { Mode I SERR } \\
G_{1} \\
\left(10^{-6} \mathrm{~N} / \mathrm{mm}^{2}\right)\end{array}$ & $\begin{array}{c}\text { Mode II SERR } \\
G_{11} \\
\left(10^{-6} \mathrm{~N} / \mathrm{mm}^{2}\right)\end{array}$ & $\begin{array}{c}\text { SERR by VCC } \\
G_{1}+G_{11} \\
\left(10^{-5} \mathrm{~N} / \mathrm{mm}^{2}\right)\end{array}$ & $\begin{array}{c}\text { Compliance } \\
C \\
\left(10^{-2} \mathrm{~N} / \mathrm{mm}\right)\end{array}$ & $\begin{array}{c}\text { Compliance } \\
\text { difference } \Delta C \\
\left(10^{-8} \mathrm{~N} / \mathrm{mm}\right)\end{array}$ & $\begin{array}{c}\text { SERR by CD } \\
(\Delta C / \Delta a) \\
\left(10^{-6} \mathrm{~N} / \mathrm{mm}^{2}\right)\end{array}$ \\
\hline$t=t_{0}$ & 8.40932 & 0.50848 & 0.89178 & $2 \cdot 72414235$ & $1 \cdot 01229$ & 0.89120 \\
\hline$t_{1}$ & $6 \cdot 80867$ & $4 \cdot 47039$ & $1 \cdot 12791$ & $2 \cdot 74457009$ & $1 \cdot 28118$ & $1 \cdot 12792$ \\
\hline$t_{2}$ & $6 \cdot 27000$ & $5 \cdot 25721$ & $1 \cdot 15272$ & $2 \cdot 77668030$ & $1 \cdot 30879$ & $1 \cdot 15223$ \\
\hline$i_{3}$ & $5 \cdot 89610$ & $5 \cdot 47117$ & $1 \cdot 13673$ & $2 \cdot 82712080$ & $1 \cdot 29135$ & $1 \cdot 13687$ \\
\hline$t_{4}$ & $5 \cdot 58005$ & 5.59210 & $1 \cdot 11722$ & 2.90630401 & $1 \cdot 27171$ & $1 \cdot 11958$ \\
\hline
\end{tabular}

Note: $\Delta a=0.00056794 \mathrm{~mm}$ is the incremental delamination growth for both VCC and CD approaches. 


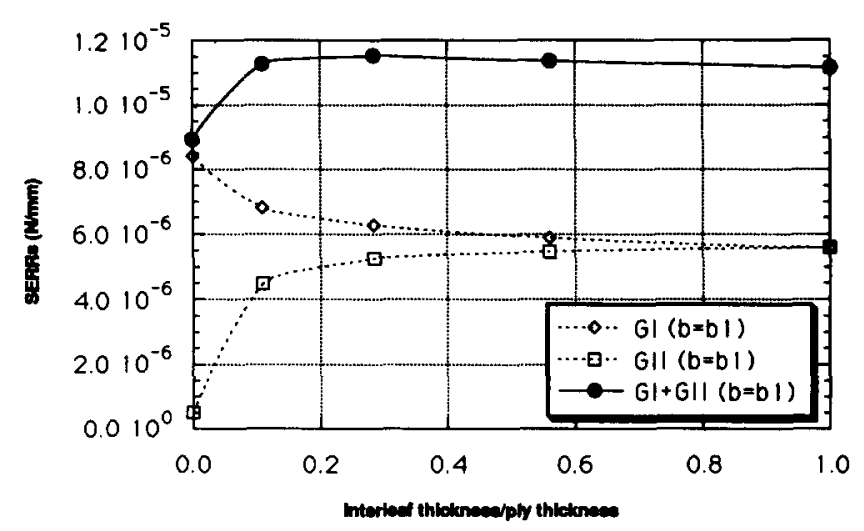

(a)

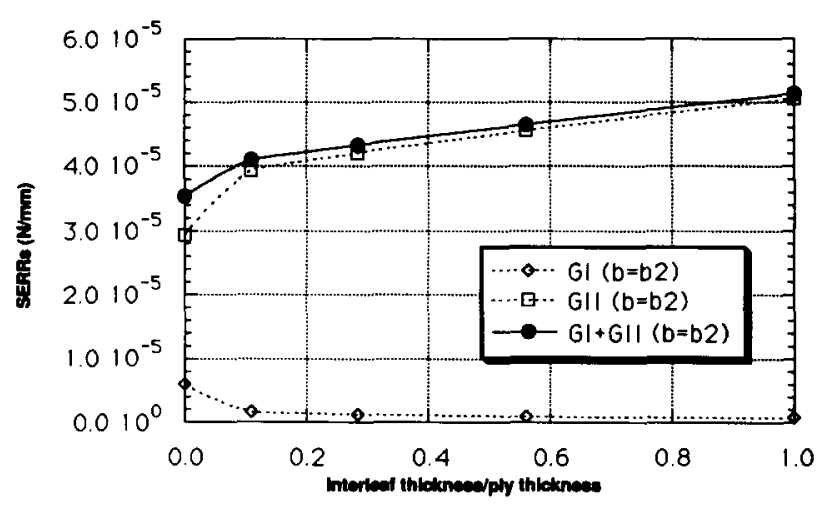

(b)

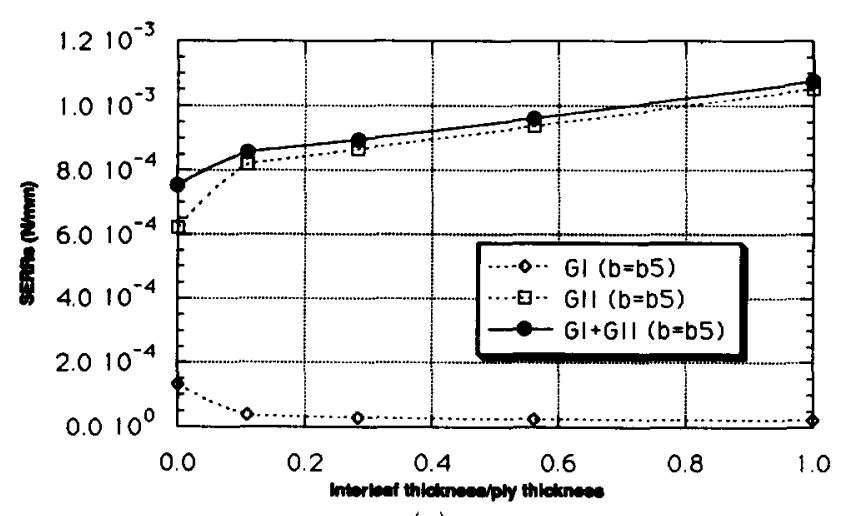

(c)

Fig. 16. Effect of interleaves on SERRs (ply thickness $0.125 \mathrm{~mm}$ ).

that for a given delamination length, embedding interleaves in the thickness range investigated only marginally alters the stress concentration. In fact, thicker interleaves slightly increases the stress concentration pertinent to the assumed manner of crack propagation, which is not welcome if examined purely from a structural perspective.

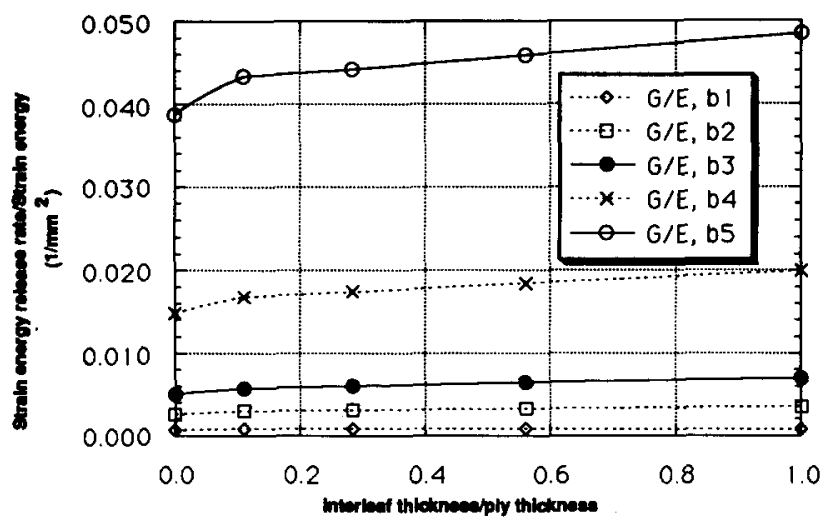

Fig. 17. Effect of interleaves on ratio of SERR and strain energy.

\section{DISCUSSION}

\subsection{Transition of fracture mode with delamination propagation}

It has been demonstrated that severe throughthickness normal stress exists at the tips of the intraply crack before delamination. This is in agreement with the result in Ref. 3, where a vertical intraply crack is assumed. The very existence of this stress renders the involvement of mode I SERR. The mode I SERR is seen to decrease relative to the mode II component as Fig. 14 shows. Further, associating this with Fig. 11, it is obvious that the mode I component diminishes extremely rapidly with the growth of the delamination.

In previous studies, ${ }^{4,5}$ the damage process has been modeled solely by delamination propagation without the involvement of the intraply crack. It was concluded that delamination propagation is always a mode II fracture event, regardless of the position of the delamination in the beam thickness direction. This is a result of the constraint of continuity of deflection and cross-sectional rotation at the delamination front and the symmetry line (equivalent to lines $|x|=b$ and $x=a$ in the current problem, see Fig. 4). In the present modeling, it appears that the intraply crack brings in a local disturbance in stress distribution near its tips, but this diminishes as soon as the delamination propagates away. The correlation of the predictions of these two models is under investigation.

In the study of transverse impact damage of composite laminates, generally mode II delamination toughness appears to correlate with the extent of damage more linearly than mode I toughness does. ${ }^{14}$ The present result seems to suggest that although the damage process does 
involve mode I fracture, it is nonetheless dominated by mode II fracture. It is perhaps worth noting that such a simplified beam model is by nature incapable of fully explaining what happens in a three-dimensional laminate damage situation. For example, in the transverse impact problem, tensile failure near the back surface of the laminate frequently occurs, which invalidates the assumption of rotational continuity of the crosssections mentioned above. As a result, the problem changes to the first scenario of interaction of intraply crack and delamination (Fig. 1(b)) which, as summarized in the Introduction, is a mode I fracture dominated failure.

\subsection{Role of interleaves in delamination fracture}

The use of the SERR/SE ratio effectively eliminates the influence of the applied load and highlights the energy releasing characteristic of a structure. It is seen from the result that the soft interleaves do not reduce the stress concentration at the delamination front. It is therefore clear that any benefit in impact damage tolerance from incorporating interleaves must stem from the intrinsic high toughness of the interleaves themselves. In this sense, the interleaf technology is a materials improvement strategy rather than a structural optimization.

A further implication of the use of the SERR/ $S E$ ratio is in the evaluation of impact damage tolerance of laminates interleaved to different extents. In practical fabrication, the number of plies of prepregs in a laminate is normally maintained, while interleaves of different thickness are embedded. This results in laminates of different total thickness, making it difficult to evaluate the impact damage tolerance on the basis of incident impact energy per laminate. ${ }^{15}$ It appears more logical to follow the concept of the SERR/SE ratio here, and evaluate damage on an absorbed energy per unit stored strain energy basis.

\section{CONCLUSIONS}

A model study of damage development in a composite laminate beam under concentrated transverse loading has been carried out. Attention has been given to the interaction between intraply crack and delamination. Influence of soft interleaves is investigated in conjunction with this nodel study.
The numerical results reveal that whether the interleaves exist or not, mode I delamination driving force is larger than that of mode II at the onset of delamination by the intraply crack. With the appearance of delamination at either tip of the intraply crack, the influence of mode I fracture component decreases rapidly. After a limited length of delamination propagation is established, the mode II delamination driving force outweighs its counterpart. Therefore, on the whole, the situation studied is essentially a mode II dominated damage process.

The ratio of SERR/SE has been used to evaluate structures with different interleaf thickness. The results based on this parameter suggest that overall, interleaves do not alleviate the fracture driving forces pertinent to delamination growth. It is thus concluded that any benefit from interleaves is entirely attributable to their intrinsic high toughness.

\section{ACKNOWLEDGEMENT}

Financial support from the Dow Chemical Company is gratefully acknowledged.

\section{REFERENCES}

1. Murri, G. B. \& Guynn, E. G., Analysis of delamination growth from matrix cracks in laminates subjected to bending loads. In Composite Materials: Testing and Design (8th Conf.) ed. J. D. Whitcomb. ASTM STP 972, American Society for Testing and Materials, Philadelphia, PA, 1988, pp. 322-39.

2. Sun, C. T. \& Manoharan, M. G., Growth of delamination cracks due to bending in $\left[90_{5} / 0_{5} / 90_{5}\right]$ laminates. Comp. Sci. \& Technol., 34 (1989) 365-77.

3. Choi, H. Y., Downs, R. J. \& Chang, F.-K., A new approach toward understanding damage mechanisms and mechanics of laminated composite due to lowvelocity impact. J. Comp. Laminates, 25 (1991) 992-1032.

4. Shi, Y. \& Hull, D., Fracture of delaminated unidirectional composite beams. J. Comp. Mater., 26 (1992) 2172-95.

5. Shi, Y., Barely visible impact damage in polymer composite laminates. PhD thesis, University of Cambridge, UK, 1991.

6. Evans, R. E. \& Masters, J. E., A new generation of epoxy composites for primary structural applications: Materials and mechanics. In Toughened Composites, ed. N. J. Johnston. ASTM STP 937, American Society for Testing and Materials, Philadelphia, PA, 1987, pp. 413-36.

7. Odagiri, N., T800H/3900-2 toughened epoxy prepreg system: Toughening concept and mechanism. In Proc 6th Technical Conf. of the American Society for Composites. Technomic Publishing, 1991, pp. 43-52. 
8. Hoisington, M. A. \& Seferis, J. C., Process-structureproperty relationships for layered structured composites. In Proc. 6th Conf. of the American Society for Composites. Technomic Publishing, 1991, pp. 53-62.

9. Rechak, S. \& Sun, C. T., Optimal use of adhesive layers in reducing impact damage in composite laminates. $J$. Reinforced Plastics \& Composites, 9 (1990) 569-82.

10. Masters, J. E., Correlation of impact and delamination resistance in interleafed laminates. In Proc. ICCM VI and ECCM II, ed. F. L. Matthews, N. C. R. Buskell, J. M. Hodgkinson \& J. Morton. Elsevier, London, 1987, pp. 3.96-3.107.

11. Aksoy, A. \& Carlsson, L. A., Interlaminar shear fracture of interleaved graphite/epoxy composites. Comp. Sci. \& Technol., 43 (1992) 55-69.
12. Aksoy, A. \& Carlsson, L. A., Crack tip yield zone estimates in mode II interlaminar fracture of interleaved composites. Engng Fracture Mech., 39 (1991) 525-34.

13. Rybicki, E. F. \& Kanninen, M. F., A finite element calculation of stress intensity factors by a modified crack closure integral. Eng. Fracture Mech., 9 (1977) 931-8.

14. Carlsson, L. A. \& Gillespie, J. W., Mode II interlaminar fracture of composites. In Application of Fracture Mechanics to Composites, ed. K. Friedrich. Elsevier Science Publishers, 1989.

15. Advanced Composites Compression Tests, BSS 7260 (B). Boeing Commercial Airplane Company, Seattle, WA, 1986. 